\title{
Caracterización de especies del género Malassezia en pacientes con dermitis seborreica y en controles
}

\author{
Elizabeth Rendic $0^{1}$, Cristina Díaz J ${ }^{2 a}$, Félix Fich $\mathbf{S}^{1}$. \\ Presence of the yeast Malassezia in \\ patients with seborrheic dermatitis \\ and subjects without skin lesions
}

Background: The yeast Malassezia spp has an established etiological role in pityriasis versicolor, folliculitis, systemic infections and onychomycosis. Aim: To assess the presence of Malassezia spp in patients with seborrheic dermatitis (SD), to find a correlation between Malassezia spp count and the severity of the disease and to compare the prevalence of the different Malassezia species in SD patients and subjects without skin lesions. Patients and methods: Scrapings of the face from 81 patients with SD (69 males) and 79 subjects (54 males) without skin lesions were obtained for a direct microscope examination and yeast culture. Results: The yeast Malassezia was found in $76 \%$ of SD patients and in $82 \%$ of subjects without skin lesions. There was a positive correlation between the number of yeasts found on direct examination and the clinical severity of lesions in SD patients. Although this correlation was statistically significant ( $\mathrm{p}=0.046)$, the degree of association $(\mathrm{rho}=0.22)$ was weak. Fifty Malassezia species were identified. M globosa was found in $67 \%$ of SD patients, followed by M furfur and M sympodialis, each present in $16.5 \%$ of the SD patients. In subjects without skin lesions, the most prevalent species were M globosa (77\%), followed by M sympodialis (12\%), M slooffiae (7\%) and M furfur (4\%). Conclusions: The presence of the yeast Malassezia is not associated with the presence of skin lesions (Rev Méd Chile 2003; 131: 1295-300).

(Key Words: Dermatitis, seborrheic; Malassezia; Yeasts)

Recibido el 5 de marzo, 2003. Aceptado en versión corregida el 1 de octubre, 2003.

${ }^{1}$ Servicio de Dermatología. Hospital Clínico San Borja-Arriarán.

${ }^{2}$ Departamento de Microbiología y Micología. Facultad de Medicina. Universidad de Chile. aTecnólogo Médico.

$\mathrm{E}^{\mathrm{n}}$ 1874, Malassez, fisiólogo e histólogo francés, describió por primera vez, levaduras en escamas de lesiones de pitiriasis simple ${ }^{1,2}$. En 1889, el término genérico Malassezia fue utilizado por Baillon en honor a Malassez ${ }^{3,4}$ para denominar hongos dimórficos observados en lesiones de pitiriasis versicolor e identifica a M furfur como la primera especie de ese género. En 1904, Sa-

Correspondencia a: Dra. Elizabeth Rendic. Agustinas 814, oficina 310. Santiago. Fono-fax: 2782852.

E mail: erendic@hotmail.com bouraud propuso el género Pityrosporum para clasificar las levaduras aisladas en cultivos de escamas del cuero cabelludo.

Se inició la coexistencia de dos sistemas taxonómicos: Malassezia y Pityrosporum y se incorporaron nuevas denominaciones, lo que aumentó la confusión y controversia, todo esto favorecido por la variable morfología y dificultad de cultivo de las levaduras ${ }^{5,6}$.

En 1996, Guého, Midgley y Guillot revisaron la taxonomía del género Malassezia con estudios morfológicos, ultraestructurales, fisiológicos y de biología 
molecular logrando identificar 4 nuevas especies de Malassezia: $\mathrm{M}$ globosa, $\mathrm{M}$ restricta, $\mathrm{M}$ obtusa y $\mathrm{M}$ slooffiae $^{7}$, las que se agregaron a la M sympodialis incorporada en 1990 por Simmons y Guého ${ }^{8}$. Es así como dentro de la hasta ahora considerada M furfur sensu lato se han logrado determinar 6 especies genéticamente distintas, las que comparten ser lípidodependientes, sin crecimiento en agar Sabouraud: M furfur sensu stricto, $\mathrm{M}$ sympodialis, $\mathrm{M}$ globosa, $\mathrm{M}$ obtusa, Mrestricta y Mslooffiae, lista a la que se sumó la M pachydermatis ${ }^{9,10}$ que sí es capaz de crecer en medios de rutina. Por lo tanto, el género Malassezia quedó integrado por estas 7 especies ${ }^{4,11,14}$, abandonándose la denominación Pityrosporum.

Malassezia spp se ha asociado a numerosas patologías quedando bien establecido su rol etiológico en pitiriasis versicolor, foliculitis, infecciones sistémicas, onicomicosis ${ }^{15,19}$. También se ha implicado a Msympodiales en gatillar formas graves de acné neonatal ${ }^{20}$ y como agente en otitis extema malig$n^{21}$. Igualmente este agente se ha asociado a dermitis seborreica (DS) en adultos s $^{4,22,24,25}$, aunque su relación patogénica es discutida ${ }^{26,30}$. Su rol en DS infantil también es motivo de discusión ${ }^{31,35}$. Considerando esta información nos pareció importante aportar a este tema planteándonos como objetivos:

1. Determinar la presencia de Malassezia spp en un grupo de pacientes con DS y en un grupo control.

2. Determinar si existe correlación entre el recuento de Malassezia spp y la severidad del cuadro.

3. Comparar la prevalencia de las distintas especies de Malassezia en el grupo de pacientes con DS y el grupo control.

\section{MATERIAL Y MÉTODO}

Se examinaron 160 pacientes mayores de 18 años que informadamente aceptaron participar en el estudio y que no habían recibido antimicóticos orales desde al menos un mes, ni tópicos desde 15 días antes de la toma de muestra. Todos los pacientes fueron examinados por el mismo médico para mantener un criterio uniforme.

$\mathrm{Al}$ grupo control ingresaron pacientes sin clínica de DS.

Ingresaron al grupo DS aquellos pacientes con lesiones en cara o cuero cabelludo clínicamente compatibles con este cuadro y se definió la siguiente escala:

$0=$ Sin lesión.

1 = Leve: descamación o eritema escaso.

2 = Moderada: eritema y descamación en regular cantidad.

3 = Severa: eritema y descamación abundantes.

Se obtuvieron muestras de todos los pacientes examinados por medio de raspado con hoja de bisturí estéril. En el caso de pacientes DS se eligieron las zonas más comprometidas en cara 0 cuero cabelludo y en los pacientes del grupo control se tomaron muestras desde la zona de implante del cuero cabelludo.

1. Examen directo microscópico: se realizó en las escamas obtenidas que se tiñeron con azul de metileno para hacer recuento de levaduras por campo microscópico, aplicándose una escala arbitraria:

$0=$ Sin levaduras por campo.

$(+) \quad=1$ a 4 levaduras por campo.

$(++)=5$ a 10 levaduras por campo.

$(+++)=$ Sobre 11 levaduras por campo.

La micromorfología también se consideró en la identificación, ya que puede ser de gran utilidad, como en el caso de la típica forma de la $\mathrm{M}$ globosa $^{36}$. En otras especies, como la $\mathrm{M}$ furfur, puede tener formas variables y la característica forma de botellita puede presentar variaciones esféricas, ovoides o cilíndricas, difíciles de reconocer y cuya interpretación es dependiente del examinador.

2. Cultivo: las escamas obtenidas se sembraron en la superficie de un medio de Dixon modificado (3,6\% extracto de malta, $0,6 \%$ peptona, $2,0 \%$ bilis de buey, $1 \%$ Tween 40, 0,2\% glicerol, 0,2\% ácido oleico, 1,2\% agar pH6)37 y se incubaron a $32^{\circ} \mathrm{C}$ por 7 días.

3. Identificación: las colonias obtenidas fueron sometidas a las siguientes pruebas fisiológicas: reacción de catalasa, desdoblamiento de la esculina y capacidad de utilizar Tween 20, 40, 60, 80 como única fuente de lípidos ${ }^{4,37}$. Se siguió el flujograma de trabajo graficado en la Figura $1^{37}$.

Reacción de catalasa: se determinó aplicando una gota de agua oxigenada de 10 volúmenes en una preparación extemporánea del cultivo en un portaobjetos. El resultado es positivo $(+)$ si se forman burbujas de gas. 


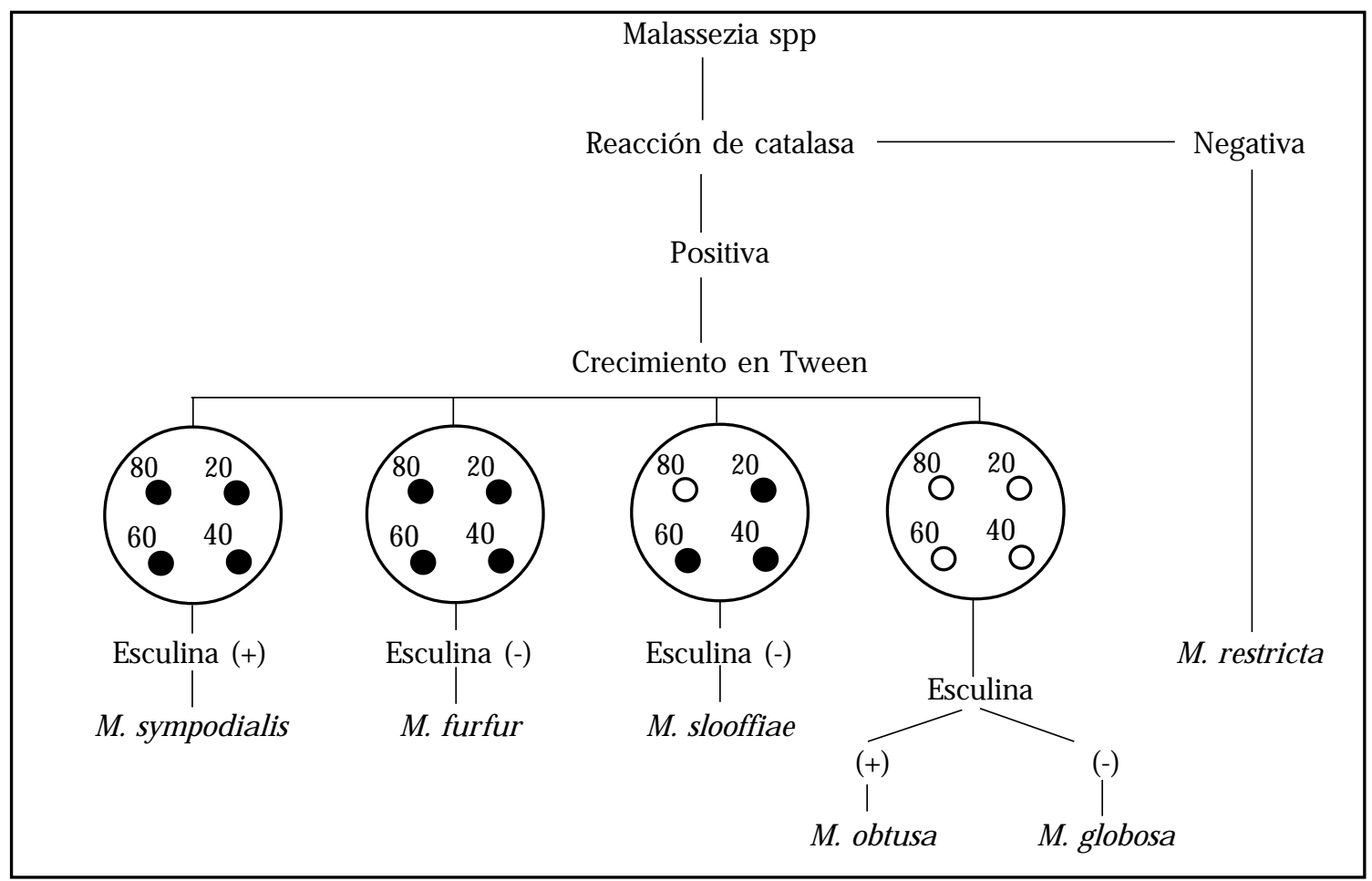

FiguRA 1. Flujograma de trabajo.

Desdoblamiento de esculina: se cultivaron levaduras en un medio con esculina. La reacción positiva (+) corresponde al viraje a negro de la esculina al desdoblarse ésta en esculitina y glucosa.

Crecimiento en Tween: a $16 \mathrm{ml}$ de Sabouraud dextrosa agar estéril se agregó una suspensión de las levaduras en estudio en $1 \mathrm{ml}$ de agua destilada estéril. La suspensión se aplicó en placa de Petri de $9 \mathrm{~cm}$ de diámetro; una vez solidificado el medio se realizaron 4 orificios de $2 \mathrm{~mm}$ y se llenaron con Tween 20, 40, 60 y 80, respectivamente. Se incubó la placa a $32^{\circ} \mathrm{C}$ por una semana, después de lo cual se examinaron con lupa las zonas de desarrollo o inhibición.

4. Análisis estadístico: se usó la prueba de $\chi^{2}$ para variables nominales (presencia-ausencia de levaduras) con un nivel de significación de $p \leq 0,05$. También se exploró si existía algún grado de asociación entre cantidad de levaduras en el examen directo y severidad clínica de la DS. Dado que ambos parámetros están expresados en variables ordinales se usó la asociación de Spearman.

\section{RESULTADOS}

De 160 pacientes estudiados, 81 corresponden al grupo DS de cara (69 hombres y 12 mujeres). Clínicamente, 32 presentaban DS leve (40\%), 26 DS moderada (32\%) y 23 DS severa (28\%).

Se definió presencia de Malassezia como examen directo $(+)$ o cultivo (+). Según se observa en la Tabla 1 encontramos presencia de Malassezia spp en $76,5 \%$ de los pacientes del grupo DS y en $82 \%$ del grupo control. Se obtuvo un $\chi^{2}$ de 0,8 con $\mathrm{p}=0,37$ (no significativo).

En la Tabla 2 se constata que no existen diferencias significativas entre los resultados del total grupo DS y grupo control. Sin embargo, se observa que recuentos altos (+++) son más frecuentes en DS severa (13\%) que en DS leve (3\%) 0 en control (5\%). Asimismo, recuentos (-) en DS leve (37\%) y control (31\%) son más frecuentes que en DS severa (17\%). Al aplicar la prueba de correlación de Spearman entre recuentos y grado de severidad clínica en el grupo DS se obtiene rho de 0,22 que tiene asociada una p de 0,046. 
Del total de levaduras aisladas, en 50 se identificaron las especies cuya presencia fue la siguiente: $36 \mathrm{M}$ globosa (72\%), $7 \mathrm{M}$ sympodialis (14\%), 5 M furfur (10\%) y 2 M slooffiae (4\%). No se aplicaron pruebas estadísticas a estos resultados por tratarse de una muestra pequeña. En la Tabla 3 observamos que M globosa fue la especie prevalente en todos los grupos, la segunda en frecuencia fue Msympodialis que presenta una leve ventaja en grupo DS con 16,5\% en relación al grupo control con 12\%. No se aisló M slooffiae en grupo DS, pero sí en grupo control se encontró en 7\%. M furfur se encontró con bastante mayor frecuencia en el grupo DS (16,5\%) que en el control (4\%).

\section{Discusión}

El estudio de levaduras del género Malassezia ha sido un tema de gran confusión y controversia. Varios reportes han avanzado en aclarar esto y confirman la redefinición del género y sus 7 especies $^{7,11,14,37}$. Sin embargo, la ecología y patogenicidad de cada especie es aún tema que requiere mayores estudios.

En este estudio no se encontró diferencia significativa entre la presencia de Malassezia spp -directo o cultivo (+)- en el grupo DS y el grupo control $(p=0,37)$ (Tabla 1$)$.
Tabla 1. Presencia de M alassezia según grupos estudiados

\begin{tabular}{|c|c|c|c|c|c|}
\hline & \multicolumn{2}{|c|}{ Presencia } & \multicolumn{2}{|c|}{ Ausencia } & \multirow[t]{2}{*}{ Total } \\
\hline & $\mathrm{n}$ & $\%$ & $\mathrm{n}$ & $\%$ & \\
\hline Grupo DS & 62 & 76,5 & 19 & 23,5 & 81 \\
\hline Control & 65 & 82 & 14 & 18 & 79 \\
\hline
\end{tabular}

$\chi^{2}=0,8 \quad p=0,37$

Considerando la severidad clínica y el número de levaduras por campo microscópico, no hay diferencias significativas entre los recuentos encontrados en grupo DS y grupo control. Estos resultados concuerdan con los de Bergbrant ${ }^{24,38}$. En nuestro estudio se constata un grado de correlación positiva (rho y p) entre número de levaduras encontradas en el examen microscópico directo y la severidad clínica en el grupo DS (Tabla 2), lo que si bien es significativo, el grado de asociación (rho $=0,22)$ es débil y desde el punto de vista clínico probablemente no tiene mayor significación.

En relación a las especies identificadas (Tabla 3), los hallazgos coinciden en gran parte con los de Makimura ${ }^{39}$, quien encontró M furfur en pacientes con DS, pitiriasis versicolor y dermitis atópica; encontró M sympodialis en pacientes con las mis-

Tabla 2. Recuento de levadura según clínica

\begin{tabular}{|c|c|c|c|c|c|c|c|c|c|}
\hline Recuentos & $\mathrm{n}$ & $\%$ & $\mathrm{n}$ & $\%$ & & $\%$ & & & $\begin{array}{l}\text { Total } \\
100 \%\end{array}$ \\
\hline DS leve & 12 & 37 & 14 & 44 & 5 & 16 & 1 & 3 & 32 \\
\hline DS moderada & 5 & 19 & 14 & 54 & 7 & 27 & - & 0 & 26 \\
\hline DS severa & 4 & 17 & 11 & 48 & 5 & 22 & 3 & 13 & 23 \\
\hline Total DS & 21 & 26 & 39 & 48 & 17 & 21 & 4 & 5 & 81 \\
\hline Control & 24 & 31 & 35 & 44 & 16 & 20 & 4 & 5 & 79 \\
\hline
\end{tabular}

Tabla 3. C aracterización de las especies de M alassezia según grupo de pacientes

\begin{tabular}{|lrrrrc|}
\hline & M globosa & M sympodialis & M furfur & M slooffiae & Total \\
& & & & \\
\hline DS leve & $5(72 \%)$ & $1(14 \%)$ & $1(14 \%)$ & - & 7 \\
DS moderada & $7(78 \%)$ & $2(22 \%)$ & $3(37,5 \%)$ & - & 9 \\
DS severa & $4(50 \%)$ & $1(12,5 \%)$ & $4(16,5 \%)$ & - & 8 \\
Total & $16(67 \%)$ & $4(16,5 \%)$ & $1(4 \%)$ & $2(7 \%)$ & 24 \\
Control & $20(77 \%)$ & $3(12 \%)$ & & \\
\hline
\end{tabular}


mas patologías como en sanos y M slooffiae en otitis media crónica y controles sanos. Sin embargo, no da cuenta de la identificación de M globosa.

Por su parte, Crespo ${ }^{36}$ encontró M globosa y M restricta como especies predominantes en DS. Sin embargo, en este estudio no identificamos la especie M restricta ni M obtusa, esta última parece estar presente en piel humana en casos excepcionales. Asimismo este autor comunica el hallazgo de M globosa en lesiones de DS y pitiriasis versicolor; de Msympodialis en piel sana y también en DS y M furfur sensu stricto, que parece ser menos frecuente, pero se ha aislado de lesiones de DS.

Algunos autores ${ }^{40}$ plantean que una alta densidad de levaduras puede presentarse sin lesiones de piel, situación que nosotros encontramos en $5 \%$ de los controles (Tabla 2) y postulan que la patogenicidad de Malassezia podría estar más relacionada con la especie involucrada que con su densidad. En nuestro trabajo, de las 8 especies identificadas en DS severa, 4 correspondieron a Mglobosa, 1 a M sympodialis y 3 a M furfur sensu stricto. Esta última especie la encontramos sólo en 1 de 7 aislamientos

\section{REFERENCIAS}

1. Malassez L. Note sur le champignon du pityriasis simple. Arch Physiol 1874; 2: 451-64.

2. HeRnÁndeZ-MoLna JM. Revisión bibliográfica sobre Malassezia (Pityrosporum): Taxonomía y su importancia. Rev Iberoam Micol 1993; 10: 24-8.

3. BaiLon H. Traité de Botanique Mèdicale Cryptogramique. Paris: O Doin 1889; 234-9.

4. Guéno E, Boekhout T, Ashbee HR, Guilot J, Van Belkum A, Faergemann J. The role of Malassezia species in the ecology of human skin as pathogens. Med Mycol 1998; 36 Sup 1: 220-9.

5. Aspiroz MC, Moreno LA, Rubio MC. Taxonomía de M furfur: Estado de la cuestión. Rev Iberoam Micol 1997; 14: 147-9.

6. Pereiro M. Situación actual de las infecciones por Malassezia. Piel 1999; 14: 76-87.

7. Guého E, Midgley G, GuiLot J. The genus Malassezia with description of four new species. Antonie van Leeuwenhoek 1996; 69: 337-55.

8. Simmons RB, Guéro E. A new species of Malassezia. Mycol Res 1990; 1146-9.

9. Guiшот J, Bond R. Malassezia pachydermatis: a review. Med Micol 1999; 37: 295-306.

10. WeIDMAn FD. Exfoliative dermatitis in the Indian rhinoceros (Rhinoceros unicornis) with en el grupo DS leve, en ninguno en DS moderada y en 1 de los 26 aislamientos del grupo control. Futuros trabajos podrían corroborar si M furfur sensu stricto es realmente más patógena que otras especies. A su vez, M slooffiae sólo la encontramos en el grupo de controles sanos.

Podemos concluir que la presencia de Malassezia spp no se correlaciona con la presencia de patología, ni tampoco, en forma categórica, el número de levaduras con la severidad de la DS. La especie más frecuentemente identificada en los dos grupos estudiados corresponde a M globosa.

El gran aporte a la nueva taxonomía del género Malassezia de Ghého, Midgley y Guillot abre una nueva línea de trabajo en este tema tan controversial y con resultados tan dispares en la literatura. ¿Cuál es el papel real que juegan las diferentes especies de Malassezia como factores gatillantes o coadyuvantes en la patología dermatológica?, chay especies más patógenas que otras?, ¿cuál es el exacto mecanismo de acción de los antimicóticos tópicos en la DS?, son preguntas que ameritan nuevas investigaciones.

description of a new species: Pityrosporum pachydermatis. In: Report of the Laboratory and Museum of Comparative Pathology of the Zoological Society of Philadelphia. Fox H ed. Zoological Society of Philadelphia, PA 1925; 36-44.

11. Gupta AK, Конц Y, Summerbell RC. Molecular differentiation of seven Malassezia species. J Clin Microbiol 2000; 38: 1969-75.

12. Boekhout T, Kamp M, GuÉHo E. Molecular typing of Malassezia species with PFGE and RAPD. Med Mycol 1998; 36: 365-72.

13. Mayser P, Haze P, Papavassilis C, Pickel M, Gruender $\mathrm{K}$, GuÉHo E. Differentiation of Malassezia species: selectivity of cremophor EL, castor oil and ricinoleic acid for M furfur. Br J Dermatol Aug 1997; 137: 208-13.

14. Aspiroz C, Moreno LA, Rezusta A, Rubio C. Differentiation of three biotypes of Malassezia species on human normal skin correspondence with $\mathrm{M}$ globosa, M sympodialis and M restricta. Mycopathologia 1999; 145: 69-74.

15. FAERGEMANN J. Pityrosporum yeast - what's new? Mycoses 1997; 40 Suppl 1: 29-32.

16. Crespo V, Ojeda A, Crespo A, Sánchez F, Guého E. Mycology of Pityriasis versicolor. J Mycol Med 1999; 9: 143-8. 
17. Schmid A. Malassezia furfur: a fungus belonging to the physiological skin flora and its relevance in skin disorders. Cutis Jan 1997; 59: 21-4.

18. Silva V, Moreno Ga, Zaror L, De Oliveira E, Fischman $O$. Isolation of Malassezia furfur from patients with onychomycosis. J Med Vet Mycol 1997; 35: 73-4.

19. Civila E, Conti-Díaz A, Vignale R, Caleggari L. Onixis por Malassezia (Pityrosporum) ovalis. Med Cut I. LA Vol X/1882; 343-6.

20. Niamba P, Weill FX, Sarlangue J, Labreze C, Couprie $\mathrm{B}$, TAIEH A. Is common neonatal cephalic pustulosis (neonatal acne) triggered by Malassezia sympodialis? Arch Dermatol 1998; 134: 995-8.

21. Chai FC, Auret K, Christiansen K, Yuen PW, Gardam D. Malignant otitis externa caused by Malassezia sympodialis. Head Neck 2000; 22: 87-9.

22. Faergemann J, Jones JC, HetTler O, Loria Y. Pityrosporum ovale (Malassezia furfur) as the causative agent of seborrheic dermatitis: new treatment options. Br J Dermatol 1996; 134 Suppl 46: 12-5 discussion 38.

23. Heid E, Laplanche G. Mycoses métropolitaines en Encyclopédie Médico-Chirurgicale. Ed Techniques Paris-France; 12320; A10-2, 1984; page 2.

24. Bergbrant M, Faergemann J. The role of $P$ ovale in Sebormeic Dermatitis. Semin Dermatol 1990; 9: 262-8.

25. BERGBRANT IM. Sebomeic Dermatitis and Pityrosporum Yeasts. Curr Top Med Mycol 1995; 6: 95-112.

26. Parry M, Sharpe G. Seborrheic dermatitis is not caused by an altered immune response to Malassezia yeasts. Br J Dermatol 1998; 139: 254-63.

27. Neuber K, Kroger S, Gruseck E, Abeck D, Ring J. Effects of Pityrosporum ovale on proliferation, immunoglobulin (IgA, G,M) synthesis and cytokine (I1-2, Il-10, IFN gamma) production of peripheral blood mononuclear cells from patients with sebormeic dermatitis. Arc Dermatol Res 1996; 288: 532-6.

28. Silva V, Fischman O, Pires de Camargo Z. Humoral immune response to $M$ furfur in patients with pityriasis versicolor and seborrheic dermatitis. Mycopathol 1997; 139: 79-85.

29. Bergbrant IM, Johansson S, Robbins D, Scheynius A, FAERGEMANN J, SoderSTROM T. An immunological study in patients with seborrheic dermatitis. Clin Exp Dermatol 1991; 16: 331-8.

30. Kesavan S, Walters CE, Holuand KT, Ingham E. The effects of Malassezia on pro-inflammatory cytokine production by human peripheral blood mononuclear cells in vitro. Med Micol 1998; 36: 97-106.

31. Tolesson A, Frithz A, Stenlund K. Malassezia furfur in Infantile Seborrheic Dermatitis. Pediatr Dermatol 1997; 6: 423-5.
32. Ruiz Maldonado R, López-Martínez R, Pérez Chavarría EL, Rocío Castanon L, Tamayo L. Pityrosporum ovale in infantil seborrheic dermatitis. Pediatr Dermatol 1989; 6: 16-20.

33. Broberg A, FAERGEMANN J. Infantile seborrheic dermatitis and Pityrosporum ovale. Br J Dermatol 1989; 120: 359-62.

34. Tolesson A, Frithz A, Stenlund K. Malassezia furfur in infantile seborrheic dermatitis. Pediatr Dermatol 1997; 14: 423-5.

35. Zegri E, Silva S, Oroz J, Prenzel I. Pityrosporum ovale en cuero cabelludo: estudio piloto en población sana de Santiago. Rev Ped (Santiago) 1992; 36: 72-6.

36. CRespo V, Ojeda A, Vera A, Crespo A, Sánchez F. Malassezia globosa as the causative agent of pityriasis versicolor. Br J Dermatol 2000; 143: 799-803.

37. GuiLot J, Guého E, Lesourd M, Midgley G, Chévrier $G$, Dupont B. Identification of Malassezia species. A practical approach. J Mycol Med 1996; 6: 103-10.

38. BeRgBRANT IM. Seborrheic Dermatitis y P ovale: cultural, inmunological and clinical studies. Acta Dermatol Venereologica, S 1991; 167: 36.

39. Makimura $K$, Tamura $Y$, Kudo M, Uchida $K$, Saito $H$, Yamaguchi $H$. Species identification and strain typing of Malassezia species stock strains and clinical isolates based on the DNA sequences of nuclear ribosomal internal transcribed spacer 1 regions. J Med Microbiol 2000; 49: 29-35.

40. Pechere M, Krischer J, Remondat C, Bertrand C, Treluu L, Saurat JH. Malassezia spp carriage in patients with seborrheic dermatitis. J Dermatol 1999; 26: 558-61.

\section{Agradecimientos}

Al Dr. Vicente Crespo Erchiga, jefe del Servicio de Dermatología del Hospital Civil CH Carlos Haya de Málaga, España, por su inagotable paciencia y generosidad con las infinitas interrogantes que nos surgían en el desarrollo de este trabajo.

A la Dra. Eveline Guého, de la Unidad de Micología del Instituto Pasteur, por su amabilidad al enviarnos material de gran ayuda para nuestro estudio.

Al Prof. Dr. Leandro Biagini Alarcón. Director del Centro de Epidemiología Clínica de la Facultad de Medicina de la Universidad de Chile, quien nos regaló valiosas horas para asesorarnos en la aplicación de las pruebas estadísticas pertinentes.

Al Servicio de Anatomía Patológica del Hospital Clínico San Borja-Arriarán por facilitarnos sus equipos para microfotografías.

Al Servicio de Dermatología del Hospital Clínico San Borja-Arriarán, a cada uno de los colegas que compartían sus pacientes para hacer posible este trabajo. 